\title{
Using Hamiltonians to Model Saturation in Space Vector Representations of AC Electrical Machines
}

\author{
Duro Basic ${ }^{1}$, Al Kassem Jebai ${ }^{2}$, François Malrait ${ }^{1}$, Philippe Martin ${ }^{2}$, and \\ Pierre Rouchon ${ }^{2}$ \\ ${ }^{1}$ Schneider Electric, STIE, 33, rue André Blanchet, 27120 Pacy-sur Eure, France. \\ 2 Mines ParisTech, Centre Automatique et Systèmes, Mathématiques et Systèmes, 60 \\ Bd Saint-Michel, 75272 Paris cedex 06, France.
}

\begin{abstract}
An Hamiltonian formulation with complex fluxes and currents is proposed. This formulation is derived from a recent Lagrangian formulation with complex electrical quantities. The complexification process avoids the usual separation into real and imaginary parts and notably simplifies modeling issues. Simple modifications of the magnetic energy underlying standard $(\alpha, \beta)$ models yield new $(\alpha, \beta)$ models describing machines with magnetic saturation and saliency. We prove that the usual expression of the electro-mechanical torque (wedge product of fluxes and currents) is related to a rotational invariance characterizing sinusoidal machines.
\end{abstract}

\section{Introduction}

In [1] a Lagrangian formulation with complex currents and fluxes is proposed. In this paper we develop the Hamiltonian counterpart only sketched in [1]. For three-phase electrical machines we recall the usual model linear in fluxes, currents and voltages, and give its Hamiltonian formulation based on magnetic energies depending quadratically on fluxes. We then propose a modification of the usual magnetic energies in order to take into account magnetic saturation. We prove that if these additional terms preserve the rotational invariance of the usual magnetic energies, then the resulting electro-magnetic torque always admits the usual form and is thus still proportional to the imaginary part of the product of complex conjugate of fluxes with stator currents.

Section 2 is devoted to permanent-magnet machines. In subsection 2.1 we present the Hamiltonian formulation of the usual model with saliency effects. In subsection 2.2 we introduce a class of saturation models and prove that, if we just replace in the usual model the constant inductances by inductances depending on the flux level, the resulting model does not admit in general a magnetic energy and thus is not correct from a physical ground. For sinusoidal machines where the magnetic energy is invariant with respect to the choice of angle origin, we prove in subsection 2.3 that the usual formula giving the electromagnetic torque as a wedge product between the flux and current remains valid 
even in the presence of saliency and magnetic saturation. Section 3 is devoted to induction machines. In subsection 3.1 we present the Hamiltonian formulation of the usual model. In subsection 3.2 we introduce a class of saturation models. For machines with sinusoidally wound phases where the magnetic energy is invariant with respect to the choice of angle origin, we prove in subsection 3.3 that the usual formula giving the electro-magnetic torque as a wedge product between the flux and current remains valid even in the presence of magnetic saturation. In section 4 we suggest some further developments.

The authors acknowledge John Chiasson for interesting discussions and precious comments.

\section{Permanent-magnet machines}

\subsection{Hamiltonian modeling}

In the $(\alpha, \beta)$ frame (total power invariant transformation), the usual dynamic equations read (see, e.g., $[2,4])$ :

$$
\left\{\begin{array}{l}
\frac{d}{d t}(J \dot{\theta})=n_{p} \Im\left(\left(\lambda \imath_{s}^{*}+\bar{\phi} e^{-\jmath n_{p} \theta}-\mu \imath_{s} e^{-2 \jmath n_{p} \theta}\right) \imath_{s}\right)-\tau_{L} \\
\frac{d}{d t}\left(\lambda \imath_{s}+\bar{\phi} e^{\jmath n_{p} \theta}-\mu \imath_{s}^{*} e^{2 \jmath n_{p} \theta}\right)=u_{s}-R_{s} \imath_{s}
\end{array}\right.
$$

where

- ${ }^{*}$ stands for complex-conjugation, $\Im$ means imaginary part, $\jmath=\sqrt{-1}$ and $n_{p}$ is the number of pairs of poles.

$-\theta$ is the rotor mechanical angle, $J$ and $\tau_{L}$ are the inertia and load torque, respectively.

$-\imath_{s} \in \mathbb{C}$ is the stator current, $u_{s} \in \mathbb{C}$ the stator voltage.

$-\lambda=\left(L_{d}+L_{q}\right) / 2$ and $\mu=\left(L_{q}-L_{d}\right) / 2$ (inductances $L_{d}>0$ and $L_{q}>0$, saliency when $\left.L_{d} \neq L_{q}\right)$.

- The constant $\bar{\phi}>0$ represents the rotor flux due to the permanent magnets.

It is proved in [1] that (1) admits the following Hamiltonian formulation

$$
\frac{d}{d t}(J \dot{\theta})=-\frac{\partial \mathcal{H}_{m}}{\partial \theta}-\tau_{L}, \quad \frac{d}{d t} \phi_{s}=u_{s}-R_{s} \imath_{s}, \quad \imath_{s}=2 \frac{\partial \mathcal{H}_{m}}{\partial \phi_{s}^{*}}
$$

where the magnetic energy $\mathcal{H}_{m}$

$$
\begin{aligned}
\mathcal{H}_{m}\left(\phi_{s}, \phi_{s}^{*}, \theta\right) & =\frac{1}{2 L_{d}}\left(\Re\left(\phi_{s} e^{-\jmath n_{p} \theta}\right)-\bar{\phi}\right)^{2}+\frac{1}{2 L_{q}}\left(\Im\left(\phi_{s} e^{-\jmath n_{p} \theta}\right)\right)^{2} \\
& =\frac{1}{2 L_{d}}\left(\frac{\phi_{s} e^{-\jmath n_{p} \theta}+\phi_{s}^{*} e^{\jmath n_{p} \theta}}{2}-\bar{\phi}\right)^{2}+\frac{1}{2 L_{q}}\left(\frac{\phi_{s} e^{-\jmath n_{p} \theta}-\phi_{s}^{*} e^{\jmath n_{p} \theta}}{2 \jmath}\right)^{2} \\
& =\frac{1}{8 L_{d}}\left(\phi_{s} e^{-\jmath n_{p} \theta}+\phi_{s}^{*} e^{\jmath n_{p} \theta}-2 \bar{\phi}\right)^{2}-\frac{1}{8 L_{q}}\left(\phi_{s} e^{-\jmath n_{p} \theta}-\phi_{s}^{*} e^{\jmath n_{p} \theta}\right)^{2}
\end{aligned}
$$

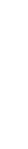


where the rotor angle $\theta$, the stator flux $\phi_{s}$ and its complex conjugate $\phi_{s}^{*}$ are considered independent variables when computing the partial derivatives of $\mathcal{H}_{m}$. In particular, $\imath_{s}=2 \frac{\partial \mathcal{H}_{m}}{\partial \phi_{s}^{*}}$ reads

$$
\begin{aligned}
\imath_{s}=\frac{e^{\jmath n_{p} \theta}}{2 L_{d}}\left(\phi_{s} e^{-\jmath n_{p} \theta}\right. & \left.+\phi_{s}^{*} e^{\jmath n_{p} \theta}-2 \bar{\phi}\right)+\frac{e^{\jmath n_{p} \theta}}{2 L_{q}}\left(\phi_{s} e^{-\jmath n_{p} \theta}-\phi_{s}^{*} e^{\jmath n_{p} \theta}\right) \\
& =\left(\frac{1}{2 L_{d}}+\frac{1}{2 L_{q}}\right) \phi_{s}-\frac{1}{L_{d}} \bar{\phi} e^{\jmath n_{p} \theta}+\left(\frac{1}{2 L_{d}}-\frac{1}{2 L_{q}}\right) \phi_{s}^{*} e^{2 \jmath n_{p} \theta} .
\end{aligned}
$$

Inverting this relation we recover the usual relation between $\phi_{s}$ and the stator current

$$
\phi_{s}=\lambda \imath_{s}+\bar{\phi} e^{\jmath n_{p} \theta}-\mu \imath_{s}^{*} e^{2 \jmath n_{p} \theta} .
$$

\subsection{Magnetic saturation}

To take into account magnetic saturation, we keep the structure equations (2) and modify the magnetic energy $\mathcal{H}_{m}$ given in (3). For obvious physical reasons, $L_{d}$ and $L_{q}$ should be decreasing functions of $\left|\phi_{s}\right|^{2}$. The simplest magnetic saturation model will be given by setting

$$
\mathcal{H}_{m}\left(\phi_{s}, \phi_{s}^{*}, \theta\right)=\frac{S_{d}\left(\left|\phi_{s}\right|^{2}\right)}{\bar{L}_{d}}\left(\Re\left(\phi_{s} e^{-\jmath n_{p} \theta}\right)-\bar{\phi}\right)^{2}+\frac{S_{q}\left(\left|\phi_{s}\right|^{2}\right)}{\bar{L}_{q}}\left(\Im\left(\phi_{s} e^{-\jmath n_{p} \theta}\right)\right)^{2}
$$

where the saturation functions $S_{d}$ and $S_{q}$ are increasing function of $\left|\phi_{s}\right|^{2}$ with $S_{d}(0)=S_{q}(0)=1$ and where $\bar{L}_{d}$ and $\bar{L}_{q}$ are the unsaturated values of $L_{d}$ and $L_{q}$ (low stator currents). The saturation model we propose is then given by (2) with this modified Hamiltonian.

Using this Hamiltonian formulation to define the relationships between $\imath_{s}, \phi_{s}$ and $\tau_{e m}$ as in (2) automatically maintains energy conservation. This conservation results from the fact that mixed partial derivatives are independent of order,

$$
\frac{\partial^{2} \mathcal{H}_{m}}{\partial \theta \partial \phi_{s}^{*}}=\frac{\partial^{2} \mathcal{H}_{m}}{\partial \phi_{s}^{*} \partial \theta} .
$$

This implies

$$
-2 \frac{\partial \tau_{e m}}{\partial \phi_{s}^{*}}=\frac{\partial \imath_{s}}{\partial \theta}
$$

where $\tau_{e m}$ and $\imath_{s}$ are considered as function of the independent variables $\phi_{s}, \phi_{s}^{*}$ and $\theta$.

On the other hand, an incorrect but seemingly "natural" way to include saturation in the usual $(\alpha, \beta)$ model

$$
\begin{aligned}
\frac{d}{d t}\left(J \frac{d}{d t} \theta\right) & =n_{p} \Im\left(\phi_{s}^{*} \imath_{s}\right)-\tau_{L} \\
\frac{d}{d t} \phi_{s} & =u_{s}-R_{s} \imath_{s} \\
\imath_{s} & =\left(\frac{1}{2 L_{d}}+\frac{1}{2 L_{q}}\right) \phi_{s}+\left(\frac{1}{2 L_{d}}-\frac{1}{2 L_{q}}\right) \phi_{s}^{*} e^{2 \jmath n_{p} \theta}-\frac{\bar{\phi}}{L_{d}} e^{\jmath n_{p} \theta}
\end{aligned}
$$


consists in taking $L_{d}$ and $L_{q}$ as function of $\rho^{2}=\phi_{s} \phi_{s}^{*}$, without changing the formula for the electro-magnetic torque. If we proceed like this we get

$\tau_{e m}=\frac{n_{p}}{2 \jmath}\left(\left(\frac{1}{2 L_{d}}-\frac{1}{2 L_{q}}\right)\left(\left(\phi_{s}^{*}\right)^{2} e^{2 \jmath n_{p} \theta}-\left(\phi_{s}\right)^{2} e^{-2 \jmath n_{p} \theta}\right)-\left(\frac{\bar{\phi}}{L_{d}}\right)\left(\phi_{s}^{*} e^{\jmath n_{p} \theta}-\phi_{s} e^{-\jmath n_{p} \theta}\right)\right)$

where $L_{d}$ and $L_{q}$ depend on $\rho^{2}=\left|\phi_{s}\right|^{2}$ Then some computations give

$$
\begin{array}{r}
-2 \frac{\partial \tau_{e m}}{\partial \phi_{s}^{*}}-\frac{\partial \imath_{s}}{\partial \theta}=\jmath n_{p}\left(\frac{d\left(\frac{1}{2 L_{d}}-\frac{1}{2 L_{q}}\right)}{d \rho^{2}}\left(\left(\phi_{s}^{*}\right)^{2} e^{2 \jmath n_{p} \theta}-\left(\phi_{s}\right)^{2} e^{-2 \jmath n_{p} \theta}\right)\right. \\
\left.-\frac{d\left(\frac{\bar{\phi}}{L_{d}}\right)}{d \rho^{2}}\left(\phi_{s}^{*} e^{\jmath n_{p} \theta}-\phi_{s} e^{-\jmath n_{p} \theta}\right)\right) \phi_{s} .
\end{array}
$$

Thus such modeling does not in general respect the commutation condition $-2 \frac{\partial \tau_{e m}}{\partial \phi_{s}^{*}}=\frac{\partial \imath_{s}}{\partial \theta}$ : no magnetic energy exists for such non-physical models. The correct current relationships include additional terms with derivatives of the functions $S_{d}$ and $S_{q}$ :

$$
\begin{aligned}
\imath_{s}=\left(\frac{1}{2 L_{d}}\right. & \left.+\frac{1}{2 L_{q}}\right) \phi_{s}+\left(\frac{1}{2 L_{d}}-\frac{1}{2 L_{q}}\right) \phi_{s}^{*} e^{2 \jmath n_{p} \theta}-\frac{\bar{\phi}}{L_{d}} e^{\jmath n_{p} \theta} \\
& +\frac{S_{d}^{\prime}\left(\left|\phi_{s}\right|^{2}\right)}{2 \bar{L}_{d}} \phi_{s}\left(\Re\left(\phi_{s} e^{-\jmath n_{p} \theta}\right)-\bar{\phi}\right)^{2}+\frac{S_{q}^{\prime}\left(\left|\phi_{s}\right|^{2}\right)}{2 \bar{L}_{q}} \phi_{s}\left(\Im\left(\phi_{s} e^{-\jmath n_{p} \theta}\right)\right)^{2}
\end{aligned}
$$

\subsection{Sinusoidal models}

Assume that the magnetic energy $\mathcal{H}_{m}$ admits the following rotational invariance associated to sinusoidal back electro-magnetic force (bemf):

$$
\forall \phi_{s} \in \mathbb{C}, \forall \theta, \xi \in \mathbb{S}^{1}, \quad \mathcal{H}_{m}\left(e^{\jmath n_{p} \xi} \phi_{s}, e^{-\jmath n_{p} \xi} \phi_{s}^{*}, \xi+\theta\right)=\mathcal{H}_{m}\left(\phi_{s}, \phi_{s}^{*}, \theta\right) .
$$

Then with $\overline{\mathcal{H}}\left(\psi, \psi^{*}\right)=\mathcal{H}_{m}\left(\psi, \psi^{*}, 0\right), \mathcal{H}_{m}$ admits the following form

$$
\mathcal{H}_{m}\left(\phi_{s}, \phi_{s}^{*}, \theta\right) \equiv \overline{\mathcal{H}}\left(\phi_{s} e^{-\jmath n_{p} \theta}, \phi_{s}^{*} e^{\jmath n_{p} \theta}\right) .
$$

In this case

$$
\begin{aligned}
\tau_{e m}=-\frac{\partial \mathcal{H}_{m}}{\partial \theta} & =-\jmath n_{p}\left(\frac{\partial \overline{\mathcal{H}}}{\partial \psi^{*}} \phi_{s}^{*} e^{\jmath n_{p} \theta}-\frac{\partial \overline{\mathcal{H}}}{\partial \psi} \phi_{s} e^{-\jmath n_{p} \theta}\right) \\
\imath_{s} & =2 \frac{\partial \mathcal{H}_{m}}{\partial \phi_{s}^{*}}=2 e^{\jmath n_{p} \theta} \frac{\partial \overline{\mathcal{H}}}{\partial \psi^{*}} .
\end{aligned}
$$

Since $\overline{\mathcal{H}}$ is a real quantity $\imath_{s}^{*}=e^{-\jmath n_{p} \theta} \frac{\partial \overline{\mathcal{H}}}{\partial \psi}$. Thus we recover the usual formula relating the electro-magnetic torque to the flux $\phi_{s}$ and current $\imath_{s}$ :

$$
\tau_{e m}=n_{p} \frac{\phi_{s}^{*} \imath_{s}-\phi_{s} \imath_{s}^{*}}{2 \jmath}=n_{p} \Im\left(\phi_{s}^{*} \imath_{s}\right) .
$$


When $\mathcal{H}_{m}$ does not admit such rotational invariance, $\tau_{e m}$ is different from $n_{p} \Im\left(\phi_{s}^{*} \imath_{s}\right)$. Thus (4) is a direct consequence of rotational invariance. The saturation models considered in the previous subsection admit this rotational invariance and yield electro-magnetic torques satisfying (4).

A simple example of a non sinusoidal model is a machine with a trapezoidal bemf $F\left(n_{p} \theta\right)$ (a sinusoidal model corresponds to $F\left(n_{p} \theta\right)=\cos \left(n_{p} \theta\right)$ ). In this case we change the Hamiltonian in (2) by

$$
\begin{aligned}
\mathcal{H}_{m}=\frac{1}{2 L_{d}}\left(\Re \left(\phi _ { s } \left(F\left(n_{p} \theta\right)+\jmath F\left(n_{p} \theta\right.\right.\right.\right. & \left.\left.\left.\left.+\frac{\pi}{2}\right)\right)\right)-\bar{\phi}\right)^{2} \\
& +\frac{1}{2 L_{q}}\left(\Im\left(\phi_{s}\left(F\left(n_{p} \theta\right)+\jmath F\left(n_{p} \theta+\frac{\pi}{2}\right)\right)\right)\right)^{2}
\end{aligned}
$$

where $e^{-\jmath n_{p} \theta}$ in (3) is replaced by $F\left(n_{p} \theta\right)+\jmath F\left(n_{p} \theta+\frac{\pi}{2}\right)$. This Hamiltonian is not rotationally invariant.

\section{Induction machines}

\subsection{Hamiltonian modeling}

We will now proceed as for permanent-magnet machines. The standard $T$-model of an induction machine admit the following form:

$$
\left\{\begin{array}{l}
\frac{d}{d t}(J \dot{\theta})=n_{p} \Im\left(L_{m} \imath_{r}^{*} e^{-\jmath n_{p} \theta} \imath_{s}\right)-\tau_{L} \\
\frac{d}{d t}\left(L_{m}\left(\imath_{r}+\imath_{s} e^{-\jmath n_{p} \theta}\right)+L_{f r} \imath_{r}\right)=-R_{r} \imath_{r} \\
\frac{d}{d t}\left(L_{m}\left(\imath_{s}+\imath_{r} e^{\jmath n_{p} \theta}\right)+L_{f s} \imath_{s}\right)=u_{s}-R_{s} \imath_{s}
\end{array}\right.
$$

where

- $n_{p}$ is the number of pairs of poles, $\theta$ is the rotor mechanical angle, $J$ and $\tau_{L}$ are the inertia and load torque, respectively.

- $\imath_{r} \in \mathbb{C}$ is the rotor current (in the rotor frame, different from the $(d, q)$ frame),$\imath_{s} \in \mathbb{C}$ the stator current (in the stator frame, i.e. the $(\alpha, \beta)$ frame) and $u_{s} \in \mathbb{C}$ the stator voltage (in the stator frame). The stator and rotor resistances are $R_{s}>0$ and $R_{r}>0$.

- The inductances $L_{m}, L_{f r}$ and $L_{f s}$ are positive parameters with $L_{f r}, L_{f s} \ll L_{m}$.

- The stator (resp. rotor) flux is $\phi_{s}=L_{m}\left(\imath_{s}+\imath_{r} e^{j n_{p} \theta}\right)+L_{f_{s}} \imath_{s}$ (resp. $\phi_{r}=$ $\left.L_{m}\left(\imath_{r}+\imath_{s} e^{-\jmath n_{p} \theta}\right)+L_{f_{r}} \imath_{r}\right)$.

The Hamiltonian formulation proposed in [1] reads:

$$
\frac{d}{d t}(J \dot{\theta})=-\frac{\partial \mathcal{H}_{m}}{\partial \theta}-\tau_{L}, \quad \frac{d}{d t} \phi_{r}=-2 R_{r} \frac{\partial \mathcal{H}_{m}}{\partial \phi_{r}^{*}}, \quad \frac{d}{d t} \phi_{s}=u_{s}-2 R_{s} \frac{\partial \mathcal{H}_{m}}{\partial \phi_{s}^{*}}
$$


where the magnetic energy $\mathcal{H}_{m}$ now depends on $\theta$, the rotor flux $\phi_{r}$ and its complex conjugate $\phi_{r}^{*}$, the stator flux $\phi_{s}$ and its complex conjugate $\phi_{s}^{*}$. The rotor (resp. stator) current is then given by $2 \frac{\partial \mathcal{H}_{m}}{\partial \phi_{r}^{*}}$ (resp. $\left.2 \frac{\partial \mathcal{H}_{m}}{\partial \phi_{s}^{*}}\right)$. For the standard model (5), we have

$$
\mathcal{H}_{m}=\frac{1}{2 L_{f}}\left(\phi_{s}-e^{\jmath n_{p} \theta} \phi_{r}\right)\left(\phi_{s}^{*}-e^{-\jmath n_{p} \theta} \phi_{r}^{*}\right)+\frac{1}{2 L_{s}} \phi_{s} \phi_{s}^{*}+\frac{1}{2 L_{r}} \phi_{r} \phi_{r}^{*}
$$

with $L_{f}=\frac{L_{f_{s}} L_{f r}}{L_{m}}+L_{f_{s}}+L_{f r}, L_{s}=L_{f s}+\frac{L_{f_{s}}+L_{f r}}{L_{f r}} L_{m}$ and $L_{r}=L_{f r}+\frac{L_{f s}+L_{f r}}{L_{f s}} L_{m}$. Such Hamiltonian formulations based on fluxes are also named $\pi$-models whereas $T$ models based on currents correspond to Lagrangian formulations (see, e.g., [5]).

\subsection{Magnetic saturation}

As in section 2.2, we will take into account magnetic saturation, we assume that in (7), $L_{s}, L_{r}$ and $L_{f}$ are decreasing function of $\left|\phi_{s}\right|^{2}$. Then the magnetic saturation model will be given by :

$$
\frac{1}{L_{s}}=\frac{S_{s}\left(\left|\phi_{s}\right|^{2}\right)}{\bar{L}_{s}}, \quad \frac{1}{L_{r}}=\frac{S_{r}\left(\left|\phi_{s}\right|^{2}\right)}{\bar{L}_{r}}, \quad \frac{1}{L_{f}}=\frac{S_{f}\left(\left|\phi_{s}\right|^{2}\right)}{\bar{L}_{f}}
$$

where the saturation functions $S_{s}, S_{r}$ and $S_{f}$ are increasing function of $\left|\phi_{s}\right|^{2}$ with $S_{s}(0)=S_{r}(0)=S_{f}(0)=1$ and where $\bar{L}_{s}, \bar{L}_{r}$ and $\bar{L}_{f}$ are the unsaturated values of $L_{s}, L_{r}$ and $L_{f}$. The saturated Hamiltonian is then

$$
\mathcal{H}_{m}=\frac{S_{f}\left(\left|\phi_{s}\right|^{2}\right)}{2 \bar{L}_{f}}\left|\phi_{s}-e^{\jmath n_{p} \theta} \phi_{r}\right|^{2}+\frac{S_{s}\left(\left|\phi_{s}\right|^{2}\right)}{2 \bar{L}_{s}}\left|\phi_{s}\right|^{2}+\frac{S_{r}\left(\left|\phi_{s}\right|^{2}\right)}{2 \bar{L}_{r}}\left|\phi_{r}\right|^{2}
$$

With the dynamic equations then given by (6). This saturation model is the Hamiltonian counter-part of the saturation model proposed in [5].

\subsection{Sinusoidal models}

The Hamiltonian $\mathcal{H}_{m}$ here above admits the following rotational invariance associated to a sinusoidal bemf:

$$
\begin{aligned}
\forall \phi_{s} \in \mathbb{C}, \forall \theta, \xi & \mathbb{S}^{1}, \\
& \mathcal{H}_{m}\left(e^{\jmath n_{p} \xi} \phi_{s}, \phi_{r}, e^{-\jmath n_{p} \xi} \phi_{s}^{*}, \phi_{r}^{*}, \xi+\theta\right)=\mathcal{H}_{m}\left(\phi_{s}, \phi_{r}, \phi_{s}^{*}, \phi_{r}^{*}, \theta\right) .
\end{aligned}
$$

Then with $\overline{\mathcal{H}}\left(\psi_{s}, \psi_{r}, \psi_{s}^{*}, \psi_{r}^{*}\right)=\mathcal{H}_{m}\left(\psi_{s}, \psi_{r}, \psi_{s}^{*}, \psi_{r}^{*}, 0\right), \mathcal{H}_{m}$ admits the following form

$$
\mathcal{H}_{m}\left(\phi_{s}, \phi_{r}, \phi_{s}^{*}, \phi_{r}^{*}, \theta\right) \equiv \overline{\mathcal{H}}\left(e^{-\jmath n_{p} \theta} \phi_{s}, \phi_{r}, e^{\jmath n_{p} \theta} \phi_{s}^{*}, \phi_{r}^{*}\right) .
$$

In this case

$$
\tau_{e m}=-\frac{\partial \mathcal{H}_{m}}{\partial \theta}=-\jmath n_{p}\left(\frac{\partial \overline{\mathcal{H}}}{\partial \psi_{s}^{*}} \phi_{s}^{*} e^{\jmath n_{p} \theta}-\frac{\partial \overline{\mathcal{H}}}{\partial \psi_{s}} \phi_{s} e^{-\jmath n_{p} \theta}\right)
$$

Since $\imath_{s}=2 \frac{\partial \mathcal{H}_{m}}{\partial \phi_{s}^{*}}=2 e^{\jmath n_{p} \theta} \frac{\partial \overline{\mathcal{H}}}{\partial \psi_{s}^{*}}$ and $\imath_{s}^{*}=2 \frac{\partial \mathcal{H}_{m}}{\partial \phi_{s}}=2 e^{-\jmath n_{p} \theta} \frac{\partial \overline{\mathcal{H}}}{\partial \psi_{s}}$ we recover the usual formula relating the electro-magnetic torque to stator flux and current:

$$
\tau_{e m}=-\jmath n_{p} \frac{\phi_{s}^{*} \imath_{s}-\phi_{s} \imath_{s}^{*}}{2}=n_{p} \Im\left(\phi_{s}^{*} \imath_{s}\right) .
$$




\section{Concluding remarks}

It remains also to validate experimentally such magnetic-saturation models. Substantial modifications to such Hamiltonian formulation are needed to include, in parallel to magnetic-saturation, magnetic hysteresis and the associated energy losses [3].

\section{References}

1. D. Basic, F. Malrait, and P. Rouchon. Euler-Lagrange models with complex currents of three-phase electrical machines and observability issues. IEEE Trans. Automatic Control, 55(1):212-217, 2010.

2. J. Chiasson. Modeling and High Performance Control of Electric Machines. IEEE Press Series on Power Engineering. Wiley-IEEE Press, 2005.

3. E. Della Torre. Magnetic Hysteresis. IEEE Press, 1999.

4. W. Leonhard. Control of Electrical Drives. Elsevier, 1985.

5. C.R. Sullivan and S.R. Sanders. Models for induction machines with magnetic saturation of the main flux path. IEEE Trans. on Industry Applications, 31(4):907914, 1995. 